\title{
CONSAGRAR LA DISTINCIÓN, PRODUCIR LA DIFERENCIA. UNA HISTORIA DEL INSTITUTO DE HUESCA A TRAVÉS DE SUS CATEDRÁTICOS (1845-1931)
}

Por Juan Mainer Baqué. Huesca: Instituto de Estudios Altoaragoneses, 2020, 445 páginas. ISBN: 978-84-8127-305-2

Cuando en 2010 llevamos a cabo una revisión de la producción bibliográfica sobre la historia de la educación secundaria desde 1981 a 2009, comenzábamos preguntándonos hasta qué punto se podía seguir hablando, en dicha fecha, de una historia desatendida, como sucedía en la década de los ochenta, o ya, por el contrario, de una historia en auge. ${ }^{1}$ Once años después puede afirmarse que la tendencia, entonces detectada, de una progresiva atención a la historia de este nivel educativo ha seguido manteniéndose. En unos casos, siguiendo una línea de investigación - ya apuntada en la primera década del nuevo siglo-centrada en el estudio, conservación y difusión del patrimonio científico, didáctico y material de los institutos de segunda enseñanza y, sobre todo, en de las disciplinas y el currículum académico-escolar. ${ }^{2}$ En otros, se trata de síntesis generales o libros colectivos con temáticas limitadas temporal o geográficamente. ${ }^{3}$ Por supuesto, han seguido apareciendo historias

\footnotetext{
1 Antonio Viñao, «La enseñanza secundaria», en Nuevas miradas historiográficas sobre la educación en la España de los siglos XIX y XX, eds. Jean-Louis Guereña, Julio Ruiz Berrio y Alejandro Tiana Ferrer (Madrid: Ministerio de Educación, Secretaría General Técnica, 2010),107-142.

2 A título de ejemplo, dentro de una relación extensa de posibles referencias, aquí carente de sentido, menciono el libro editado, en la fase inicial del programa de I+D Ceimes, por Leoncio López-Ocón, Santiago Aragón y Mario Pedrezuela (eds.), Aulas con memoria. Ciencia, educación y patrimonio en los institutos históricos de Madrid (1837-1936) (Madrid: CEIMES, CSIC y Comunidad de Madrid, 2012).

${ }_{3}^{3}$ Por ejemplo, Guillermo Vicente y Guerrero (coord. y ed. lit.), Historia de la Enseñanza Media en Aragón (Zaragoza: Institución «Fernando el Católico», 2011), donde el autor de la obra que reseñamos ya publicó un avance de la misma, y Estudios sobre la historia de la enseñanza secundaria en
} 
específicas de determinadas instituciones docentes, públicas o privadas, editadas con motivo de la celebración de los 25, 50, 100 o más años de su creación, y escritas, por lo general, desde un talante más hagiográfico-conmemorativo, a modo de crónica, que científico, ${ }^{4}$ así como, desde una perspectiva general, sobre la red de institutos públicos. ${ }^{5}$ También el profesorado ha seguido siendo objeto, cómo no, de algún que otro trabajo, bien sobre aspectos concretos como su formación y selección o la depuración franquista del mismo, bien a partir de enfoques generales ${ }^{6}$, así como la evolución cuantitativa de su alumnado ${ }^{7}$, o aspectos específicos del alumnado femenino. ${ }^{8}$ Por último, no está de más reseñar aquí, en esta apresurada serie de referencias bibliográficas mínimas, el reciente

Aragón (Zaragoza: Institución «Fernando el Católico», 2012), o Leoncio López-Ocón, «Un esfuerzo para superar el carácter elitista de la educación secundaria: el caso de la Segunda República española (1931-1936), Resgate 29 (2021): 1-32.

${ }^{4}$ Excepción clara de dicha orientación es el libro de Raimundo Cuesta y Antonio Molpereces, Retazos, memorias y relatos del Bachillerato. El Instituto Fray Luis de León de Salamanca (1931-2009) (Salamanca: Instituto Fray Luis de León, 2010), con el que la obra que reseñamos guarda una clara relación metodológica y conceptual, pese a circunscribirse a una época anterior. Siguiendo esta línea, ya en la página inicial de la «Presentación» del libro, advierte Juan Mainer que esta historia de «su» Instituto se aleja de esa «literatura apologética, asaz indigesta, que navega entre la nostalgia panegírica y el insulso anecdotario sobre la escuela que ya fue, a mayor gloria de lo que ahora es», y rehúye tanto la «idealización del pasado» como cualquier «atisbo de planglosianismo o de conmiseración del presente» (p. 11)

${ }^{5}$ José I. Cruz, «Los institutos de segunda enseñanza. Datos sobre su implantación (1835-1936)», Educatio Siglo XXI 30 (1), (2012): 233-252, y «La extensión del bachillerato en los suburbios españoles (1956-1984). Las Secciones Filiales de Institutos», Estudios sobre educación 32 (2017): 115-134. Sobre la 'desaparición' de un buen número de institutos, a modo de castigo impuesto por su origen republicano, Antonio F. Canales Serrano, "Innecesarios a todas luces": el desmantellament de la xarxa d'instituts de la postguerra», Educació i Història 17 (2011): 187-212.

${ }^{6}$ Antonio Viñao, «Modelos de formación inicial del profesorado de educación secundaria en España (siglos XIX-XXI)», Revista Española de Educación Comparada 22 (2013): 19-37; Olegario Negrín Fajardo, «La depuración franquista del profesorado de los Institutos de Segunda Enseñanza», en La larga noche de la educación española. El sistema educativo español en la posguerra, eds. Antonio F. Canales Serrano y Amparo Gómez Rodríguez (Madrid: Biblioteca Nueva, 2015), 39-69; Raimundo Cuesta Fernández y Juan Mainer Baqué, «Guardianes de la tradición y esclavos de la rutina; historia del campo profesional de los catedráticos de instituto», Historia y Memoria de la Educación 1 (2015): 351-393, un artículo, este último, que en el que los autores avanzan y sostienen, desde una perspectiva general, algunas de las tesis y enfoques del libro que reseñamos.

7 José I. Cruz, «Oficiales, colegiados y libres. Una revisión crítica de la estadística del bachillerato en España (1930-1970)», Revista de Educación 374 (2016): 211-231. Se trata, en efecto, de una revisión crítica donde se corrige, por ejemplo, algún error del cuadro sobre la distribución del alumnado según el tipo de enseñanza, que figura en Antonio Viñao, Escuela para todos. Educación y modernidad en la España del siglo XX (Madrid: Marcial Pons Historia, 2004), 195.

${ }_{8}$ Antonio F. Canales Serrano, «Little intelectuals. Girls' academic secondary education under Francoism: projects, realities and paradoxes», Gender and Education 24 (4), (2012): 375-391. 
interés por el tema de los historiadores de la economía. En este caso, desde enfoques preferentemente estadístico-cuantitativos, no exentos de análisis cualitativos, ya se trate de trabajos sobre la formación del cuerpo de catedráticos de segunda enseñanza, la expansión de la escolarización en este nivel educativo o las desigualdades de acceso al mismo. ${ }^{9}$

Es en este contexto historiográfico, brevemente sintetizado y ejemplificado, en el que hay que situar el nuevo libro de Juan Mainer sobre el Instituto de Segunda Enseñanza de Huesca desde su creación en 1845 hasta el comienzo de la Segunda República en 1931.

\section{SÍNTESIS E INFORMACIÓN BÁSICA DE LA OBRA}

Este es el primer fruto de un estudio cronológicamente más amplio sobre uno de esos institutos de Segunda Enseñanza hoy llamados «históricos», el de Huesca, creado en 1845 a consecuencia del Plan Pidal, como todos aquellos que, ubicados en las capitales de provincia, no habían abierto sus puertas en el decenio precedente. El segundo libro, de publicación próxima, llevará por título Del elitismo a la masificación. Historia y memoria del Ramón y Cajal de Huesca y cubrirá desde 1931 hasta prácticamente el presente con especial atención a los cambios producidos en la década de los noventa del pasado siglo.

Queda claro, pues, que este primer libro abarca una buena parte de lo que el autor, como miembro del proyecto Nebraska de la Federación Icaria ${ }^{10}$ cuyas categorías conceptuales y analíticas sigue, califica de «modo de educación tradicional-elitista» (1836-1959) y, dentro del mismo, de sus dos primeras fases: la «constituyente» de «creación» (1836-1857) y «pervivencia» (1857-1900) del sistema educativo español, y la de «reformulación discursiva y reformas» o «comienzo de la transición larga» (1900-1939).11

\footnotetext{
9 Pau Insa-Sánchez, «Catedráticos in the making of the Spanish secondary education system, 18611885», Paedagogica Historica, en prensa, e "Inequality of opportunity in access to secondary education in 19th century Spain", documento de trabajo de la Asociación Española de Historia Económica (DT-AEHE), accessible en https://ideas.repec.org/p/ahe/dtaehe/2106.html, y Pau Insa y Alfonso Díez-Minguela, «Bringing education to the masses. The expansion of secondary schooling in Spain, 1857-1901», documento de trabajo (textos facilitados por los autores).

${ }^{10}$ http:///www.nebaskaria.es

11 Dichas categorías conceptuales y analíticas han sido expuestas y utilizadas en diversas ocasiones por los componentes del proyecto Nebraska. Por ejemplo, en Raimundo Cuesta, Juan Mainer y Julio Mateos, "Modos de educación y problemas de periodización histórica desde una perspectiva
} 
En suma, y por lo que a la segunda enseñanza se refiere, estamos ante un estudio centrado en una institución docente de las conocidas como "pequeñas universidades de provincias», del que fue un bachillerato elitista y androcéntrico — rasgo, este último, quebrado a partir de 1910 más legal y oficialmente que por los hechos, el currículo, o el día a día-, y - este es uno de sus rasgos más relevantes- en una «historia» del Instituto "a través de sus catedráticos», como se dice en el título de la obra.

En efecto, si el primer capítulo del libro sitúa el Instituto oscense en el «modo de educación tradicional elitista» inaugurado con la creación de la segunda enseñanza durante los años de la revolución liberal (el paso de la Universidad Sertoriana al nuevo Instituto, sus difíciles primeros años, aspectos materiales, organizativos y pedagógicos, alumnado, planes de estudio, el contexto socioeducativo, etc.), los otros tres, el resto o cuerpo central del libro, se apartan del contenido habitual en otras historias de este tipo para estructurarse en torno a 36 entradas relativas a otros tantos catedráticos distribuidos en tres grupos: «los catedráticos isabelinos, fundadores de la profesión» (19), «los catedráticos de la Restauración: la consolidación del canon socioprofesional» (8) y «los catedráticos del regeneracionismo, garantes del canon: tradición y modernización» (9). Todo ello precedido, en cada uno de los tres capítulos, de unas páginas dedicadas a la génesis, evolución, características, configuración y naturaleza del nuevo nivel educativo creado por la revolución liberal y la formación y selección del nuevo cuerpo que dio vida a los nuevos establecimientos docentes: el de catedráticos de bachillerato y, a su vera, por debajo de ese nuevo cuerpo, el de los llamados, según el momento, profesores auxiliares, adjuntos, sustitutos, etc.

Esta es, sin duda, la parte más original del libro. Como el mismo autor se encarga de destacar, no estamos ante un diccionario biográfico, aunque la estructura formalmente elegida sea la propia de un trabajo de este tipo. De ahí que cada uno de los 36 catedráticos «biografiados» vea acompañado su nombre y datos relativos al nacimiento y fallecimiento, de un breve calificativo que lo define profesional y/o ideológicamente o en relación con su posición o papel en el Instituto: desde el primero de

genealógica», en Transiciones, cambios y periodizaciones en la historia de la educación, coords. Raimundo Cuesta, Juan Mainer y Julio Mateos (Salamanca: Lulú, 2009), 41-67, y «Reformas y modos de educación en España: entre la tradición liberal y la tecnocracia», Revista Andorra 11 (2011): 16-95. 
ellos ( Bartolomé Beato Sánchez: fugaz espejismo liberal en el Instituto postsertoriano») al último ( Benigno Baratech Montes: la derecha católica y autoritaria oscense en la dirección»). O, asimismo, las diferencias existentes en cuanto a la extensión de unas y otras biografías, a causa, sobre todo, del distinto enfoque y uso que se hace de cada una de ellas: desde las simplemente descriptivas a las que sirven al autor para llevar a cabo todo tipo de consideraciones contextuales sobre el personaje, su época, su actividad académica, social y profesional o, incluso, sobre la tendencia ideológico-académico-pedagógica que representa. De ahí que, como se indica en un momento determinado, lo que va a encontrar quien lea el libro son «tres historias en una: la del Instituto, la de sus profesores y la de Huesca como escenario de la evolución del bachillerato profesional» (p. 19).

La obra se completa con varios anexos (relación, por disciplinas, de los catedráticos del Instituto oscense desde 1845 a 1990; de los sucesivos directores del Instituto desde 1845; y datos sobre el analfabetismo en la provincia de Huesca, por partidos judiciales, en 1930), una extensa bibliografía, la relación de fuentes consultadas y un siempre útil índice onomástico.

\section{NATURALEZA, CARÁCTER, OBJETIVOS}

Queda dicho: no estamos ante una historia más de un Instituto concreto de lo que hoy llamamos educación secundaria. El propósito del autor va mucho más allá. Como él mismo nos dice, se trata de:

Un ejercicio de historia social y crítica, teóricamente informada, que, tomando el Instituto como objeto de estudio, se ocupará de indagar sobre la cuestión de la educación en el marco de la conflictiva realidad social y política de nuestros siglos XIX y XX. Así pues, el primer y principalísimo propósito de este libro es contribuir a pensar la educación como un problema social y a problematizar sus instituciones como sujetos históricos: escenarios contingentes en los que se desarrollan confrontaciones y luchas por el poder (p. 13).

Más en concreto, centrando dicho objetivo general en la institución objeto de estudio, el autor añade que este libro «nació con la vocación 
de ser, por encima de todo, una historia del presente, una explicación sociogenética de los problemas de la institución escolar en la actualidad». Entre otras razones, porque solo así:

Estaremos en condiciones de problematizar y completar esa imagen complaciente y progresista del Instituto que la Huesca eterna y victoriosa de la gente de orden ha nutrido y ha hecho formar parte de un imaginario común y colectivo ocultando su lado más oscuro, que es, en suma, la cara siempre ocultada de la escuela del capitalismo (p. 19).

La consecución de dichos objetivos exige no solo un buen dominio del arte de la escritura - un rasgo apreciable en toda la obra del autor-, de cómo se escribe la historia para que «diga cosas» con claridad y precisión, es decir, sentido y profundidad. También exige, en este caso, una difícil imbricación, bien lograda, entre los enfoques macro y micro, lo estatal-nacional y lo regional-local, lo institucional y lo personal, el mundo académico y el entorno social, económico, cultural y político en que dicho mundo se inserta y del que se nutre y vive, o entre las periodizaciones socioeducativas y las político-legislativas. Esta difícil combinación de miradas amplía los temas que podrían ser objeto de un comentario específico: el peso de las antiguas instituciones —en este caso, la Universidad de Huesca - en aquellas —el nuevo Instituto- que emergen de las mismas; el lento proceso de consolidación socioprofesional del cuerpo de catedráticos de bachillerato a partir de una situación inicial de naturaleza precaria y provisional por lo que se refiere a su formación, títulos y selección; la importancia en la vida académica de los Institutos del profesorado auxiliar, sustituto o adjunto; la organización académica y el papel relevante, por competencias y representatividad, de los directores; la formación del canon curricular de la segunda enseñanza y de los distintos campos disciplinares, etc. Los temas a comentar podrían ser muchos. De todos ellos, hemos elegido, por su índole general y metodológica, la cuestión de las periodizaciones.

\section{LA CUESTIÓN DE LAS PERIODIZACIONES}

Todos los historiadores trabajamos con periodizaciones más o menos generales a las que asignamos unas denominaciones determinadas. Entre los historiadores de la educación —como en otros campos 
historiográficos - se tiende a establecer límites temporales coincidentes con fechas relevantes ligadas a cambios políticos, legislativos o político-educativos. En el caso español y en el período contemporáneo, por ejemplo, 1808, 1857, 1931, 1936, 1939, 1970, 1990, etc. Otras veces, tomamos prestadas periodizaciones políticas cuyas denominaciones - trienio liberal, sexenio democrático, segunda república, franquismo, transición, democracia...- nos sirven para adjetivar no solo un tipo determinado de educación, sino, en ocasiones, de profesorado o currículum: liberal, republicano, franquista, democrático, etc.

Es cierto que, en el fondo, el recurso a estas u otras periodizaciones plantea los mismos problemas que la determinación de límites temporales artificiales como los siglos — por ejemplo, «la educación en la España del siglo XX»— o eras — por ejemplo, «la educación en la Alta o Baja Edad Media»-, forzando, en el primer caso, la presencia de necesarias alusiones a los períodos precedente y subsiguiente, y, en el segundo, la búsqueda de las fechas clave de comienzo y final para cada una de dichos períodos o eras. En palabras de Guy Lardreau, en conversación con Georges Duby, «la cronología es absolutamente decisiva para la historia» hasta el punto, añadía, de que «no hay historia si no hay un trabajo cronológico», o sea, «más que allí donde se es capaz de establecer una cronología». Cuestión a la que Duby respondía que «lo que hace a la historia es una referencia, lo más precisa posible, a una duración. De aquí la necesidad de establecer puntos de referencia cronológicos, alrededor de los cuales se sitúan los temas». En su opinión, «el enriquecimiento de la historia ha venido, en gran parte, del hecho de que los historiadores se hayan vuelto hacia fenómenos y huellas que son difícilmente fechables».12

La cuestión, en este caso, no es pues más que un aspecto concreto de este rasgo general a toda investigación histórica, apreciable asimismo, entre otros campos historiográficos, en la historia de la economía, de la ciencia o de la cultura; es decir, en aquellos subsistemas o ámbitos sociales cuyo análisis requiere periodizaciones y cronologías no subsumibles

\footnotetext{
12 Georges Duby, Diálogo sobre la historia. Conversaciones con Guy Lardreau (Madrid, Alianza Editorial, 1988), 55-56. Sobre el particular, remito a lo dicho en Espacio y tiempo. Educación e historia (Morelia: Instituto Michoacano de Ciencias de la Educación, 1996), 39-46, donde, comentando dicha «conversación», traté la cuestión de las periodizaciones y los peligros, en este punto, tanto del contextualismo como del presentismo.
} 
sin más, en las usuales periodizaciones de índole temporal —siglos, edades, eras-o político-legislativas.

La referida tendencia o hábito generalizado a utilizar periodizaciones político-legislativas en el ámbito histórico-educativo viene siendo cuestionada, con razón, desde al menos tres enfoques, sin negar, desde luego, la repercusión mayor o menor en un sentido - aceleración del ritmo de los cambios - u otro - estancamientos o retrocesos- que los cambios político-legislativos originan en la educación:

Por un lado, por quienes, desde el concepto de cultura escolar, reconocen a los sistemas e instituciones educativas la capacidad de generar productos - disciplinas o tareas - y formas de hacer - prácticas- y pensar - mentalidades - propias e independientes, en mayor o menor grado, de dichos cambios.

Por otro, por quienes, desde el concepto de gramática de la escolaridad, u otros similares, han puesto el acento más en las continuidades, hibridaciones y adaptaciones que en los cambios o rupturas, poniendo en cuestión, además, el alcance real de las reformas educativas emprendidas desde los poderes públicos o privados, así como de la legislación, instrucciones, programas, cuestionarios o normas curriculares y materiales, tanto si son sugeridos a título orientativo al profesorado como si se le imponen.

Por último, por quienes, desde la historia social, analizan procesos socioeducativos de larga duración - alfabetización, escolarización, feminización, profesionalización docente-que dependen tanto o más de cambios socioculturales que políticos.

Es esta atención hacia los aspectos socioeducativos, económicos y culturales la que llevó en su día al grupo Nebraska a elaborar, para el análisis de la educación española desde el siglo XIX a nuestros días, un modelo de periodización basado no en los hitos político-legislativos usuales, sino en el concepto de «modo de educación» — desde el tradicional elitista al tecnocrática de masas en el que seguiríamos actualmente- con dos fases de transición «larga» (1900-1960) o «corta» (1960-1970) y sus momentos de aceleración, estancamiento, sus continuidades y rupturas. ${ }^{13}$ En

\footnotetext{
13 Para más detalle, véanse los trabajos de Raimundo Cuesta, Juan Mainer y Julio Mateos menciona-
} dos en la nota 11. En esta obra, Mainer caracteriza los «modos de educación» como «una categoría 
un seminario celebrado en el CEINCE en 2009, comenté y efectué una serie de observaciones sobre el tema. ${ }^{14}$ Aquí solo las completaré indicando que, en todo caso, los autores de este modelo de periodización nunca, como buenos historiadores, lo han interpretado y utilizado de un modo rígido e inflexible, sino como una categoría heurística a adaptar en función del tema objeto de estudio. Así lo señalé, en otros trabajos, en relación con su interpretación de las continuidades y rupturas entre la Segunda República y el franquismo o la naturaleza y ubicación temporal de la Ley General de Educación de 1970. ${ }^{15}$ En la obra que comentamos, el autor mismo recurre a adjetivaciones temporalmente políticas — catedráticos «isabelinos», "de la Restauración» o «del regeneracionismo»- o finaliza esta primera parte de la historia del Instituto oscense no en 1900 - comienzo de la «transición larga» hacia el modo de educación tecnocrático de masas- o en 1960 —fecha de inicio de la «transición corta» y final de la anterior_-, sino en una fecha claramente política: en 1931, con la proclamación de la Segunda República. Decisión que, con independencia de otras razones no dichas, justifica diciendo que en este volumen cubre desde la fundación del Instituto en 1845 «hasta el despliegue de los primeros síntomas del modo de educación tradicional elitista, que en el establecimiento oscense comenzaron a ser netamente perceptibles durante la dictadura de Primo de Rivera» (p. 19). Y ello porque fue «sin duda» en el "primer tercio» del siglo XX «cuando se anunciaron y se prefiguraron formulaciones teóricas y principiaron algunas mutaciones cuantitativas y cualitativas propias de lo que sería la racionalidad del modo de educación tecnocrático de masas» (p. 17). Mutaciones reforzadas durante la Segunda República, al menos cuantitativamente, que no serán recuperadas, en un contexto político

\footnotetext{
heurística, una especie de gran armazón conceptual o, si se prefiere, los prismáticos de alcance, que nos permite estudiar y problematizar la evolución del sistema educativo español en sus relaciones con el sistema de producción y la organización social capitalista, en la seguridad de que la historia social de la escuela es inseparable de la historia social de la burguesía. [...] Podríamos decir, en definitiva, que los modos de educación proporcionan al mismo tiempo, una herramienta de análisis y un mecanismo de periodización para abordar el estudio de los sistemas educativos» (p. 15).

14 Antonio Viñao, «Modos de educación y problemas de periodización histórica. Comentarios y observaciones", en Transiciones, cambios y periodizaciones en la historia de la educación, coords. Raimundo Cuesta, Juan Mainer y Julio Mateos (Salamanca: Lulú, 2009), 83-98.

15 Antonio Viñao, "Modos de educación y problemas de periodización histórica», 89-90, y «La Ley General de Educación de 1970. ¿Final de una etapa? ¿comienzo de otra?», en Modernización educativa y socialización política. Contenidos curriculares y manuales escolares en España durante el tardofranquismo y la Transición democrática, ed. Manuel Ferraz Lorenzo (Madrid: Universidad de La Laguna y Editorial Morata, 2020), 125-149 (referencias 127-128).
} 
e ideológico harto diferente, hasta veinte años más tarde, en la década de los sesenta. Juan Mainer deja esta parte para el segundo volumen. Un volumen que, al menos en mi caso, espero con cierta expectativa. No ya por lo que preludia o anuncia el primero, sino porque en él tendrá que abordar la historia de «su» institución, del estamento docente al que pertenece, del nivel educativo cuyas transformaciones ha sufrido y de una ciudad en la que vive. No es tarea fácil ser al mismo tiempo protagonista y espectador distanciado de lo que se narra. Sobre todo, si uno no se considera "guardián de la tradición» y «esclavo de la rutina».

\section{DISTINCIÓN Y DIFERENCIA}

El título del libro de Juan Mainer - Consagrar la distinción, producir la diferencia - evoca el ya clásico de Bourdieu sobre la «distinción» y las «bases sociales del gusto». ${ }^{16}$ Por si acaso no quedara clara dicha filiación, lo primero que se encuentra al abrirlo es la dedicatoria: «A quienes quedaron fuera». Una distinción — ser bachiller- que marcaba la diferencia entre quienes accedían y culminaban la nueva segunda enseñanza y quienes se quedaban fuera de ella. El bachillerato constituía, a la vez, una barrera y un nivel según la también clásica expresión de Edmond Goblot cuando en 1925, refiriéndose al país vecino, escribía: «Se llega a ser burgués; pero para eso es preciso, primero, llegar a ser bachiller. Cuando una familia se eleva de la clase popular a la burguesía, ello no sucede en una sola generación. Llega cuando se consigue dar a sus hijos la instrucción secundaria y hacerles pasar el bachillerato». ${ }^{17} \mathrm{O}$, como el mismo Mainer dice en relación con España:

Hasta bien entrados los años setenta ser bachiller suponía estar en posesión de un título que confería prestigio, cierta posición y posibilidades: significaba pertenecer por derecho propio a una cierta élite social. Con el título de bachiller se adquiría el trato de don, algo más que un detalle en el juego simbólico de la distinción, que expresaba las formas propias de la vieja aristocracia de sangre a las de una suerte de renovado elitismo adquirido

\footnotetext{
16 Pierre Bourdieu, La distinción. Criterio y bases sociales del gusto (Madrid: Taurus, 1988).

${ }_{17}$ Edmond Globot, La barriére et le niveau. Étude sociologique sur la bourgeoisie française moderne (Paris: PUF, 1985), 85-86.
} 
mediante el esfuerzo y el estudio. [...] los institutos, sin duda, fueron, acaso mucho más que hoy en día, instrumentos modeladores - o sancionadores-de precisas y distinguidas identidades sociales. Consagraron la distinción, produjeron la diferencia y, en consecuencia, contribuyeron a multiplicar la división (p. 14).

Queda para el anunciado libro de Mainer sobre la historia del Instituto oscense «Ramón y Cajal», desde 1931 hasta fechas recientes, ver y entender cómo y qué sucedió cuando el elitismo dio paso a la masificación, cuando el bachillerato de unos pocos pasó a ser, no sin tensiones, algún trauma y obstáculos - a trancas y barrancas - la educación secundaria para todos. Al menos, desde un punto de vista formal: la distinción y la diferencia permanecen, solo que ahora legitimadas por el perverso paraguas de la «igualdad de oportunidades». Pero esa es otra historia.

Antonio Viñao Frago

Universidad de Murcia avinao@um.es 